Check for updates

Cite this: RSC Adv., 2017, 7, 40866

\title{
Excellent stability of thicker shell CdSe@ZnS/ZnS quantum dots $\uparrow$
}

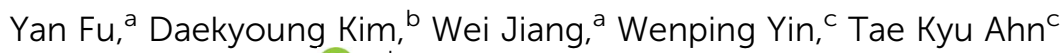 \\ and Heeyeop Chae (D)*ab
}

Significant progresses made in the performance of quantum dot (QD) light-emitting diodes have often been associated with basic changes in QD synthesis, particularly with respect to composition and structure. Here we show that the absolute photoluminescence (PL) quantum yield (QY) of QD can reach $88 \%$ with a full width of $21 \mathrm{~nm}$ at half-maximum (FWHM) when an optimal thickness is chosen for the outer shell of alloyed, graded core/shell QDs. Thicker shell QD-LEDs with a maximum current efficiency of $56.6 \mathrm{~cd} \mathrm{~A} \mathrm{~A}^{-1}$, an external quantum efficiency of $14.8 \%$, and a brightness of $62000 \mathrm{~cd} \mathrm{~m}^{-2}$ are demonstrated. Green emitting QDs of CdSe@ZnS/ZnS with this optimal thickness is also most resistant to photo-stimulated degradation by UV exposure. Only a 7\% loss in PL intensity results even after more than 400 hours of exposure to harsh conditions of $85^{\circ} \mathrm{C}$ and $85 \%$ relative humidity.

Received 22nd June 2017

Accepted 15th August 2017

DOI: 10.1039/c7ra06957j

rsc.li/rsc-advances

CdSe/CdS core-shell QDs. With an optimal outer shell thick-

\section{Introduction}

Colloidal quantum dots (QDs) have emerged as a new generation luminescent material with size-dependent and thus tunable emission properties. They have shown great potential for applications in light emitting diodes (LEDs),$^{1-4}$ solar cells, ${ }^{5,6}$ and biomarkers ${ }^{7-10}$ because of their unique optical properties such as high photoluminescence (PL) quantum yield and narrow emission profile (15-40 nm FWHM), and because of their excellent solution processability. ${ }^{11-19}$ To realize their potential, however, high quality quantum dots are needed that are stable.

An important measure of quality of light-emitting QDs is PL quantum yield, which is the ratio of the number of photons emitted over the number of ultra-violet (UV) photons absorbed. This yield is typically reduced by non-radiative recombination paths originated from surface defects ${ }^{20}$ and Förster resonance energy transfer (FRET). ${ }^{21-23}$ Stable QDs do not lose their PL intensity when they go through purification, processing for device fabrication, and device operation, which exposes the QDs to high temperature, air, moisture, and chemicals.

An effective approach for better quality and stability has been to use an outer shell that coats the inner core/shell QD. An initial attempt $\mathrm{t}^{24}$ was to utilize a double outer shell for inner

${ }^{a}$ School of Chemical Engineering, Sungkyunkwan University (SKKU), Suwon, 16419, Republic of Korea. E-mail: hchae@skku.edu

${ }^{b}$ Sungkyunk Advanced Institute of Nanotechnology (SAINT), Sungkyunkwan University (SKKU), Suwon, 16419, Republic of Korea

${ }^{c}$ Department of Energy Science, Sungkyunkwan University (SKKU), Suwon, 16419, Republic of Korea

$\dagger$ Electronic supplementary information (ESI) available. See DOI: 10.1039/c7ra06957j ness, the QY was increased to $72 \%$ and the QDs were stable even after purification and UV exposure. Different double outer shells were also utilized to improve the luminescence efficiency. ${ }^{25}$ For the simpler single outer shell that coats the inner core/shell QD, significant improvements in device performance has been achieved by alloyed cor ${ }^{20}$ and alloyed intermediate shell. ${ }^{22}$ With optimally graded, alloyed intermediate shell, an external quantum efficiency (EQE) exceeding $20 \%$ and a current efficiency higher than $80 \mathrm{~cd} \mathrm{~A}^{-1}$ have been achieved. ${ }^{26,27}$

The big strides made in the performance are the results of optimization with respect to the structure and composition of QDs, which indicates that QDs are at the core for the device performance even though there are many other materials needed for fabrication of LEDs. In this work, we simply focus on QDs to enhance the optical and physical properties of QDs. In particular, we explore the beneficial effects that can be achieved by optimizing with respect to the outer shell thickness.

\section{Experimental}

2.1 Syntheses of green CdSe@ZnS alloyed core/shell QDs (A-QDs) and CdSe@ZnS/ZnS (alloyed core/shell)/thick shell QDs (AS-QDs)

Green CdSe@ZnS alloyed core/shell QDs, or A-QDs, with a chemical composition gradient were synthesized by modifying the method described elsewhere. ${ }^{4,22,28}$ For the synthesis, cadmium acetate $\left(\mathrm{Cd}(\mathrm{acet})_{2}, 99.9 \%\right)$, zinc oxide $(\mathrm{ZnO}, 99.99 \%)$, $\mathrm{Zn}$ acetate dihydrate $\left(\mathrm{Zn}\left(\mathrm{CH}_{3} \mathrm{COO}\right)_{2} \cdot 2 \mathrm{H}_{2} \mathrm{O}\right)$, selenium (Se, 99.9\%), sulfur (S, 99.9\%), oleic acid (OA, 90\%), 1-octadecene (ODE, 90\%), trioctylphosphine (TOP, 90\%) and hexane (HPLC grade, $97.0 \%)$ were purchased from Sigma-Aldrich. Acetone 
(99.5\%) and anhydrous ethanol (99.5\%) were obtained from Daejung Chemicals and Metals Co., Ltd. All the chemicals were used as received without further purification. Typically, a mixture of $0.14 \mathrm{mmol}$ of $\mathrm{Cd}$ (acet) ${ }_{2}$ powder, $3.14 \mathrm{mmol}$ of $\mathrm{ZnO}$ powder, and $7 \mathrm{~mL}$ of OA was stirred by a magnetic bar in a $50 \mathrm{~mL}$ three-neck flask under a flow of nitrogen gas, heated to $150{ }^{\circ} \mathrm{C}$, and degassed for $30 \mathrm{~min}$. Following the addition of ODE $(15 \mathrm{~mL})$, the temperature was raised to $310^{\circ} \mathrm{C}$. At this temperature, a stock solution of $\mathrm{Se}(1.5 \mathrm{mmol})$ and $\mathrm{S}(2.5 \mathrm{mmol})$ in TOP $(3 \mathrm{~mL})$ was swiftly added into the reaction mixture. The reaction temperature was kept at $310^{\circ} \mathrm{C}$ for $10 \mathrm{~min}$ to form the quarternary CdSe@ZnS alloyed core/shell QDs. The heating mantle was then removed and cooled to room temperature in water bath and diluted with hexane and excess anhydrous ethanol to remove excess ligands and precursors by centrifugation. For fabrication of CdSe@ZnS/ZnS (alloyed core/shell)/thick shell QDs, or AS-QDs, S-ODE (1.6 mmol in $2.4 \mathrm{~mL})$ stock solution was injected continuously at $310^{\circ} \mathrm{C}$ for $12 \mathrm{~min}$ to the above reaction solution without centrifugation, after $10 \mathrm{~min}$ of reaction. $\mathrm{Zn}$ acetate dihydrate $(2 \mathrm{mmol}$ or $3 \mathrm{mmol}$ or $4 \mathrm{mmol}$ ) was dissolved in OA + ODE mixed solution to form a clear stock solution. After injecting the solution, the reaction temperature was lowered to $270{ }^{\circ} \mathrm{C}$. Then, S-TOP solution $(6 \mathrm{mmol}$ or $9 \mathrm{mmol}$ or $12 \mathrm{mmol}$ ) was added dropwise for $10 \mathrm{~min}$. The reactor temperature was maintained at the injection temperature for $20 \mathrm{~min}$. The amount of $\mathrm{Zn}$ and $\mathrm{S}$ was varied to obtain different outer ZnS-shell thickness. The centrifugation procedure of the reaction mixture was the same as for A-QDs. Finally, the precipitate was dispersed in hexane for analyses such as UV-vis, PL, TEM, and TRPL measurements, and QD film formation.

\subsection{Device fabrication}

The quantum-dot light-emitting diodes (QLEDs) were fabricated on ITO glass substrate cleaned using acetone and methyl alcohol under ultrasonication over $10 \mathrm{~min}$. ZnO NPs were spincoated at $1500 \mathrm{rpm}$ for $30 \mathrm{~s}$ onto the ITO glass substrate and baked at $160{ }^{\circ} \mathrm{C}$ for $20 \mathrm{~min}$ as the electron transport layer (ETL). The QD in the blend solvents of hexane and octane was spin coated onto the $\mathrm{ZnO}$ layer at $3000 \mathrm{rpm}$ for $30 \mathrm{~s}$ as the emitting layer (EML). PEIE buffer layer were coated with same condition, then annealing at $130{ }^{\circ} \mathrm{C}$ for $10 \mathrm{~min}$. Poly-TPD $\left(10 \mathrm{mg} \mathrm{mL}^{-1}\right)$ as hole transport layer (HTL) and $\mathrm{MoO}_{x}\left(10 \mathrm{mg} \mathrm{mL}{ }^{-1}\right)$ as hole injection layer (HIL) were also spin-coated at $3000 \mathrm{rpm}$ for $30 \mathrm{~s}$ with same annealing temperature $\left(120{ }^{\circ} \mathrm{C}\right)$, at $20 \mathrm{~min}$ and $10 \mathrm{~min}$ respectively. Aluminum (Al, $100 \mathrm{~nm}$ ) was deposited by thermal evaporation under $1 \times 10^{-5}$ Torr.

\subsection{Optical analysis}

UV-visible absorption (UV-vis) spectra and PL emission spectra were collected at room temperature with an FP-6200 spectrofluorometer and a V-630 Bio UV-vis spectrophotometer, respectively. The absolute PL quantum yield (QY) ${ }^{29}$ of the QDs was measured by QE-2100 from Otsuka Electronics Co., Ltd. We selected the $405 \mathrm{~nm}$ as the excitation wavelength for each QDs solution. The size and composition of A-, and AS-QDs were characterized with a JEM ARM 200F transmission electron microscope (TEM) and energy dispersive X-ray spectrometer (EDS). EDS spectra was averaged for hundreds of QDs from the different TEM image area. QDs dispersed in hexane were deposited from diluted solutions onto copper grids with carbon support. Wide-angle X-ray diffraction (WAXS) was performed with a Rigaku D/MAX-III diffractometer using $\mathrm{Cu}-\mathrm{K} \alpha$ radiation operating at $30 \mathrm{kV}$ and $40 \mathrm{~mA}$. Each scan took about $20 \mathrm{~min}$ with a $2 \theta$ angular range from $5^{\circ}$ to $80^{\circ}$. All measurements were performed at room temperature. Observed PL dynamics were measured using a time correlated single photon counting (TCSPC) system. The samples were excited at $405 \mathrm{~nm}$, and the measurements were made at a repetition rate of $800 \mathrm{kHz}$ at room temperature. The current density-voltage-luminance $(J-V-L)$ characteristics of the devices were measured by a source measure unit (2400, Keithley Instruments, Inc.) and a luminance meter (CS-2000, Konica Minolta Sensing, Inc.).

\subsection{Stability analysis}

The photo-oxidation experiments were performed by roomtemperature ultraviolet (UV) treatment. The PL intensity of the solid-state QDs film (spin-coated on glass, $\sim 30 \mathrm{~nm}$ ) was measured in certain time intervals followed by the PL measurements of the films during UV irradiation exposure $\left(185 \mathrm{~nm}, 254 \mathrm{~nm}, 180 \mathrm{~mW} \mathrm{\textrm {cm } ^ { - 2 }}\right.$ ) in $\mathrm{O}_{2}$ environment and $\mathrm{UV}$ irradiation exposure $\left(185 \mathrm{~nm}, 254 \mathrm{~nm}, 180 \mathrm{~mW} \mathrm{~cm}^{-2}\right)$ in air environment. Long-term thermal stability was performed by subjecting the QD films to high temperature $\left(120^{\circ} \mathrm{C}\right.$ in $\mathrm{N}_{2}$ glove box) and high humidity $\left(85^{\circ} \mathrm{C}\right.$ and $85 \%$, and $25{ }^{\circ} \mathrm{C}$ and $50 \%$ relative humidity conditions in air).

\section{Results and discussion}

Fig. 1 shows TEM images of CdSe@ZnS A- and CdSe@ZnS/ZnS AS-QDs for the same CdSe@ZnS alloyed core/shell size but with $\mathrm{ZnS}$ outer shell of different thickness. The average diameter of the A-QDs, which is shown in Fig. 1a, was $9 \pm 0.45 \mathrm{~nm}$ with no outer shell. The formation of CdSe@ZnS alloyed core/shell confirms with EDS mapping as shown in Fig. S1a and b. $\dagger$ All the Cd reacts with Se and S before any of the zinc as core of A-QDs, and a large excess of zinc reacts with Se and $S$ as outer shell of A-QDs. The diameter of AS-QDs including the outer shell in Fig. 1b-d were $12 \pm 0.45 \mathrm{~nm}, 13 \pm 0.55 \mathrm{~nm}$, and $13.6 \pm$ $0.95 \mathrm{~nm}$, respectively. To verify the composition of nanocrystals, the atoms ratio of elements in both the core and shell structure averaged over hundred QDs were determined (Fig. S1c $\dagger$ ). The size of nanocrystals become larger gradually by the increasing amounts of $\mathrm{Zn}$ and $\mathrm{S}$ after the stage of alloyed core growth. Distinct lattice spacing observable in the high resolution transmission electron microscopy (HRTEM) images except for the largest ones indicates high crystalline quality of A- and AS-QDs. No distinct interfaces could be noticeable in the QDs, which can be attributed to good mutual diffusion leading to smooth interfaces.

Shown in Fig. 2 are the UV-vis absorption and PL emission spectra of CdSe@ZnS A-QDs and CdSe@ZnS/ZnS AS-QDs as affected by the thickness of the outer shell. As the size of QDs or 


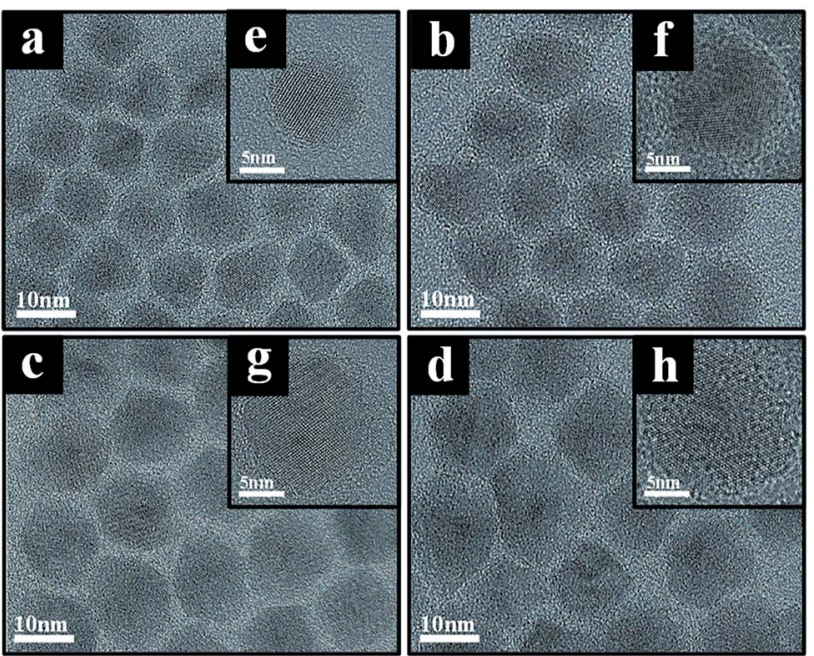

Fig. 1 TEM and HRTEM (inset) images of the CdSeaZnS A-QDs and CdSeaZnS/ZnS AS-QDs obtained by different amount of ZnS (a) CdSeaZnS A-QDs (9 nm QDs); (b) CdSeaZnS/ZnS AS-QDs (12 nm QDs, 2 mmol of ZnS); (c) CdSe (aZnS/ZnS AS-QDs (13 nm QDs, $3 \mathrm{mmol}$ of ZnS); (d) CdSe@ZnS/ZnS AS-QDs (13.6 nm QDs, $4 \mathrm{mmol}$ of ZnS).

the thickness of the outer shell increases, the first excitation peak and sharp emission peaks are gradually blue shifted ${ }^{12,19,30}$ and the absorption peak of $300-350 \mathrm{~nm}$ is increased, which is an indication of $\mathrm{ZnS}$ formation. During the growth stage of $\mathrm{ZnS}$ shell, $\mathrm{S}$ anion is richer than Zn cation (table in Fig. S1c†). Therefore, $\mathrm{S}$ atoms diffuse into the alloyed core and it leads to the formation of CdS, and the wavelength becomes blue shifted. ${ }^{31}$ The PL spectra in the inset shows that the PL intensity of CdSe@ZnS/ZnS AS-QDs is more than twice the intensity of CdSe@ZnS A-QDs, with a full width at half maximum (FWHM) of only $21 \mathrm{~nm}$. The absolute PL QY of the nanocrystals also increase from $44 \%$ for A-QDs up to $88 \%$ for AS-QDs (see Table 1).

To examine the crystallographic features of the colloidal QDs, powder X-ray diffraction (XRD) analysis was conducted and the results are shown in Fig. 3. The standard XRD patterns of the theoretical peak positions of CdSe, CdS, ZnSe and ZnS bulk crystals are also provided in the bottom of the figure. Three distinct diffraction peaks of CdSe@ZnS A-QDs are located among those of CdSe, CdS, ZnSe and ZnS bulk crystals, confirming the growth of QDs with composition gradient (CdZnSeS). The diffraction peaks of QDs with composition gradient shifts to larger angles (the standard ZnS position) gradually as the $\mathrm{Zn}$ content increases, which is consistent with the epitaxial growth of a ZnS shell onto CdSe@ZnS A-QDs. The QDs grow in all directions while maintaining the spherical shape throughout the reaction.

To gain an insight into the effects of the outer shell thickness on the photoluminescence, exciton decay is recorded for both solution and film form of QDs by a time correlated single photon counting (TCSPC) system. Fig. 4a shows that the lifetime of the QDs in solution ( $\tau_{0}$ in Table 1$)$, as represented by the time at which the intensity is reduced by an exponential factor, is greatly increased by the presence of the outer shell, the exciton lifetime increasing from $10.3 \mathrm{~ns}$ to $14.8 \mathrm{~ns}$, an increase of $\sim 44 \%$

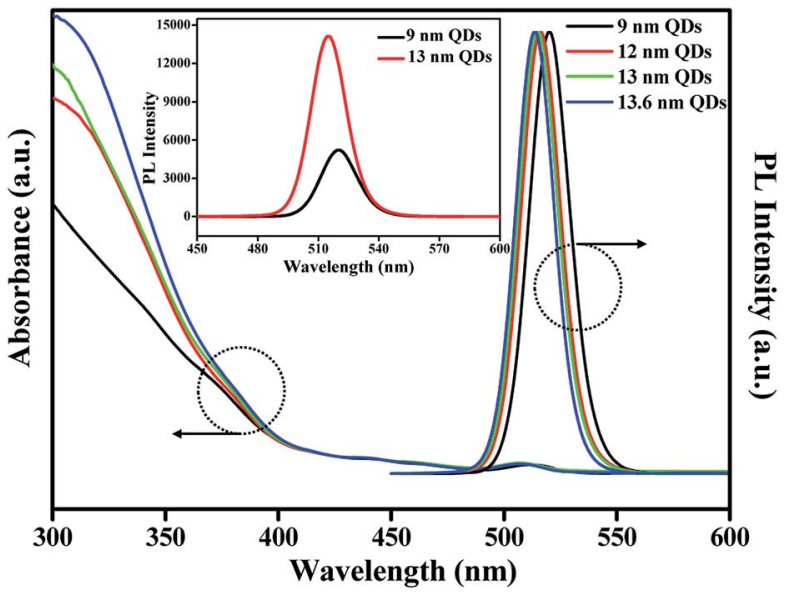

Fig. 2 UV-vis absorption and PL spectra of QDs with different outer shell thickness (inset: the PL intensity of $9 \mathrm{~nm}$ QDs and $13 \mathrm{~nm}$ QDs, respectively).

due to the reduced non-radiative recombination rate $\left(k_{\text {nrad }}\right)$ with the presence of the outer shell (Table 1). When QDs are assembled in solid film, on the other hand, the thickness affects the exciton lifetime of the QDs significantly as revealed by the lifetime $\tau_{\mathrm{f}}$ in Table 1 and the decay behavior shown in Fig. $4 \mathrm{~b}$. In the film, the QDs are packed intimately, touching one another unlike those well dispersed in solution, and the non-radiative FRET would come into play because the rate of FRET is sensitively dependent on dot-to-dot distance when the distance is less than $10 \mathrm{~nm}$. This fact explains why the lifetime is drastically reduced by 50 to $70 \%$ compared to that in the solution. However, the lifetime of the QDs in the film should progressively increase with increasing outer shell thickness up to a certain thickness by the suppression of the dot-to-dot energy transfer in solid film. ${ }^{23}$ The further increasing the shell thickness would be lead to increase non-radiative recombination of solution which are generation of the poor crystalline surface (Fig. 1h), thereby decreasing the lifetime. The optimum outer shell thickness, as shown in Table 1, is approximately $1.9 \mathrm{~nm}$, which corresponds to a QD size of $13 \mathrm{~nm}$. This result is also confirmed by the lowest non-radiative recombination rate $\left(k_{\text {nrad }}\right)^{32}$ that the QD of $13 \mathrm{~nm}$ has (Table 1$)$.

To exemplify the importance of the optimal outer shell thickness, we explored A-QDs, AS-QDs as the emitting layer in LEDs. The cross-sectional transmission electron microscopy (TEM) image of an inverted structure QLED is shown in Fig. 5a that was fabricated with layers of ITO/ZnO/QDs/ polyethylenimine ethoxylated (PEIE)/poly ( $N, N^{\prime}$-bis (4-butylphenyl)- $N, N^{\prime}$-bis (phenyl)-benzidine) (poly-TPD)/MoO $/$ /Al. All the active layers are solution-deposited except for $\mathrm{Al}$ electrode. Note that an interfacial layer of PEIE is introduced between QD layer and poly-TPD HTL. It plays multiple roles of protecting the integrity of the QD layer, which is derived from its hydrophilic property, and upshifting the valence band maximum (VBM) of QD, which arises due to the interfacial dipole property. It reduces the barrier height against hole-transport from HTL to EML, resulting in an optimized charge balance in the device. 
Table 1 Structural characteristics and optical properties as affected by size of A- and AS-QDs

\begin{tabular}{|c|c|c|c|c|c|c|c|}
\hline QDs notation & $\begin{array}{l}\text { Core radius }(r) / \text { shell } \\
\text { thickness }(H)\left(\mathrm{nm} \mathrm{nm}^{-1}\right)\end{array}$ & $\begin{array}{l}\text { Total diameter } \\
(D \pm \mathrm{Std}, \mathrm{nm})\end{array}$ & $\begin{array}{l}\text { PL peak/FWHM } \\
\left(\mathrm{nm} \mathrm{nm}^{-1}\right)\end{array}$ & PL QY (\%) & $\tau_{0}(\mathrm{~ns})$ & $\tau_{\mathrm{f}}(\mathrm{ns})$ & $k_{\text {nrad }}$ \\
\hline $9 \mathrm{~nm}$ QDs & $4.5 / 0$ & $9 \pm 0.45$ & $521 / 22$ & 44 & 10.3 & 3.4 & 0.054 \\
\hline 13 nm QDs & $4.5 / 1.9$ & $13 \pm 0.55$ & $515 / 21$ & 88 & 14.8 & 7.2 & 0.008 \\
\hline $13.6 \mathrm{~nm}$ QDs & $4.5 / 2.2$ & $13.6 \pm 0.95$ & $514 / 21$ & 82 & 12.9 & 6.4 & 0.014 \\
\hline
\end{tabular}

Fig. 5b and c give the current density-voltage-luminance $(J-V-L)$ characteristics, and current efficiency and external quantum efficiency characteristics, respectively. The figures show that the QLED based on A-QDs (9 $\mathrm{nm}$ ) yields a maximum brightness of approximately $20000 \mathrm{~cd} \mathrm{~m}^{-2}$, a current efficiency (CE) of $7.3 \mathrm{~cd} \mathrm{~A}^{-1}$, and an external quantum efficiency (EQE) of $1.9 \%$. A substantial improvement in the performance of QLEDs resulted when AS-QDs were used. With the optimal thickness of $13 \mathrm{~nm}$, in particular, the peak luminescence increased to $62700 \mathrm{~cd} \mathrm{~m}^{-2}$, the $\mathrm{CE}$ to $56.6 \mathrm{~cd} \mathrm{~A}^{-1}$, and the peak EQE to $14.8 \%$ with a turn-on voltage of $3.5 \mathrm{~V}$. These values represent a 3-fold increase in brightness, and a more than 7-fold increase in both current and external quantum efficiencies, compared with A-QDs device.

A thicker shell tends to reduce surface trap states and suppress the dot-to-dot energy transfer. Furthermore, a reduction in non-radiative Auger recombination due to a thicker shell must have contributed significantly to enhancing the device performance. ${ }^{33}$ Auger recombination is a process of nonradiative recombination due to the energy transferred to a third carrier, which decreases the luminescence of QDs. ${ }^{34}$ After the growth of the thicker shell, electron wave function is delocalized into the thick shell region, whereas the hole remains in the core by a strong confinement in the core. ${ }^{35}$ That

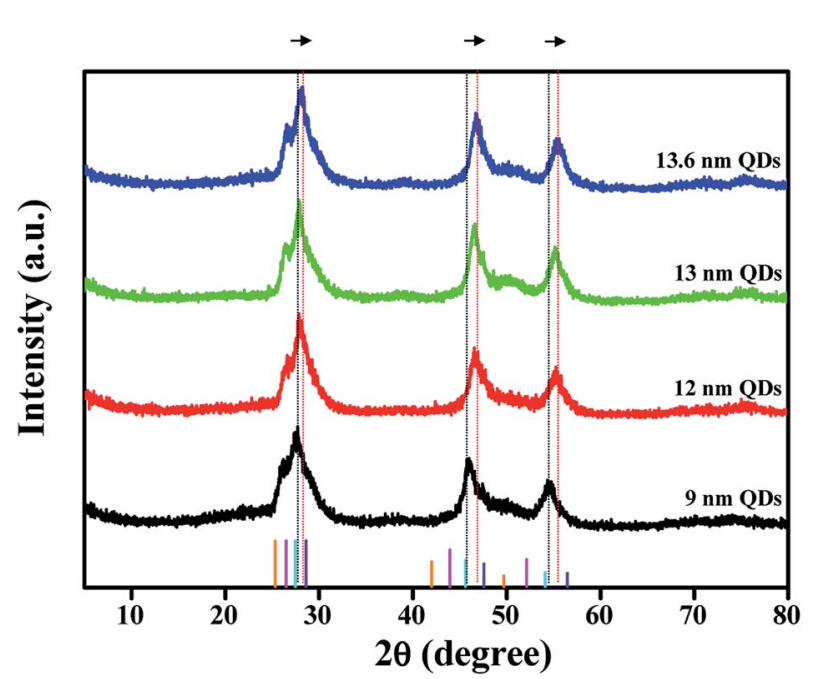

Fig. 3 Powder X-ray diffraction patterns of CdSeaZnS A-QDs, and CdSeaZnS/ZnS AS-QDs as affected by outer shell thickness. Bottom XRD peaks are the standards for CdSe (PDF\#19-0191_orange line), CdS (PDF\#10-0454_pink line), ZnSe (PDF\#80-0021_blue line) and ZnS (PDF\#65-0390_violet line). weakens the interaction of carriers by reducing the electronhole spatial overlap. Therefore, a thicker shell suppresses charging and reduces the Auger recombination efficiency, resulting in better performance of QLEDs.

In addition to the benefits accrued from the outer shell discussed above, the outer layer of $\mathrm{ZnS}$ has a lower charge density on its surface compared to $\mathrm{CdS}$, which would reduce photo-stimulated degradation of QDs. ${ }^{12}$ The photo-oxidation stability of quantum dot is important for industrial applications. To test the stability, A- and AS-QD films were subjected to UV irradiation in $\mathrm{O}_{2}$ and air filled environment. Fig. 6a and $\mathrm{S} 2 \mathrm{a} \dagger$ show the evolution of the photo-oxidation stability in terms of relative PL intensity. For the tests, the films were exposed to
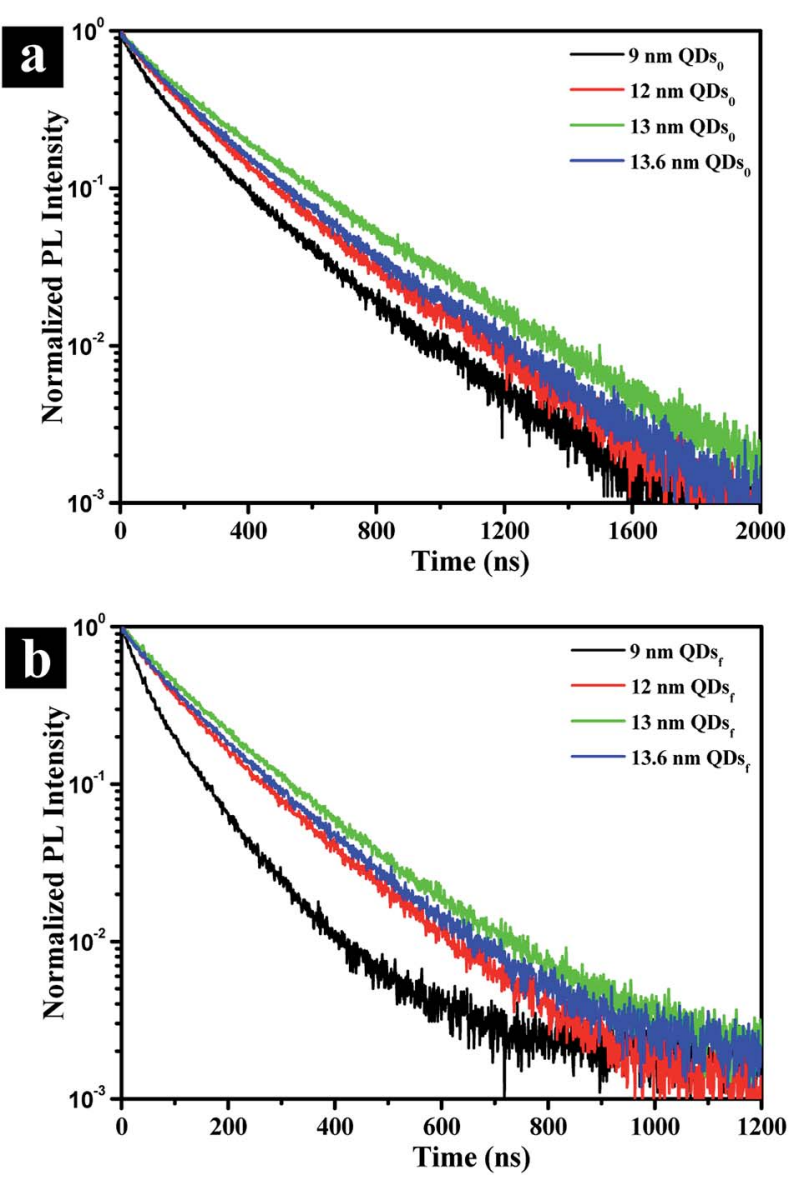

Fig. 4 Photoluminescence decay curves in solution (a) and film (b) states for CdSeaZnS A-QDs, and CdSe@ZnS/ZnS AS-QDs as affected by outer shell thickness (QDs $s_{0}$ : QDs solution; $Q D s_{f}$ : QDs film). 


\section{a}
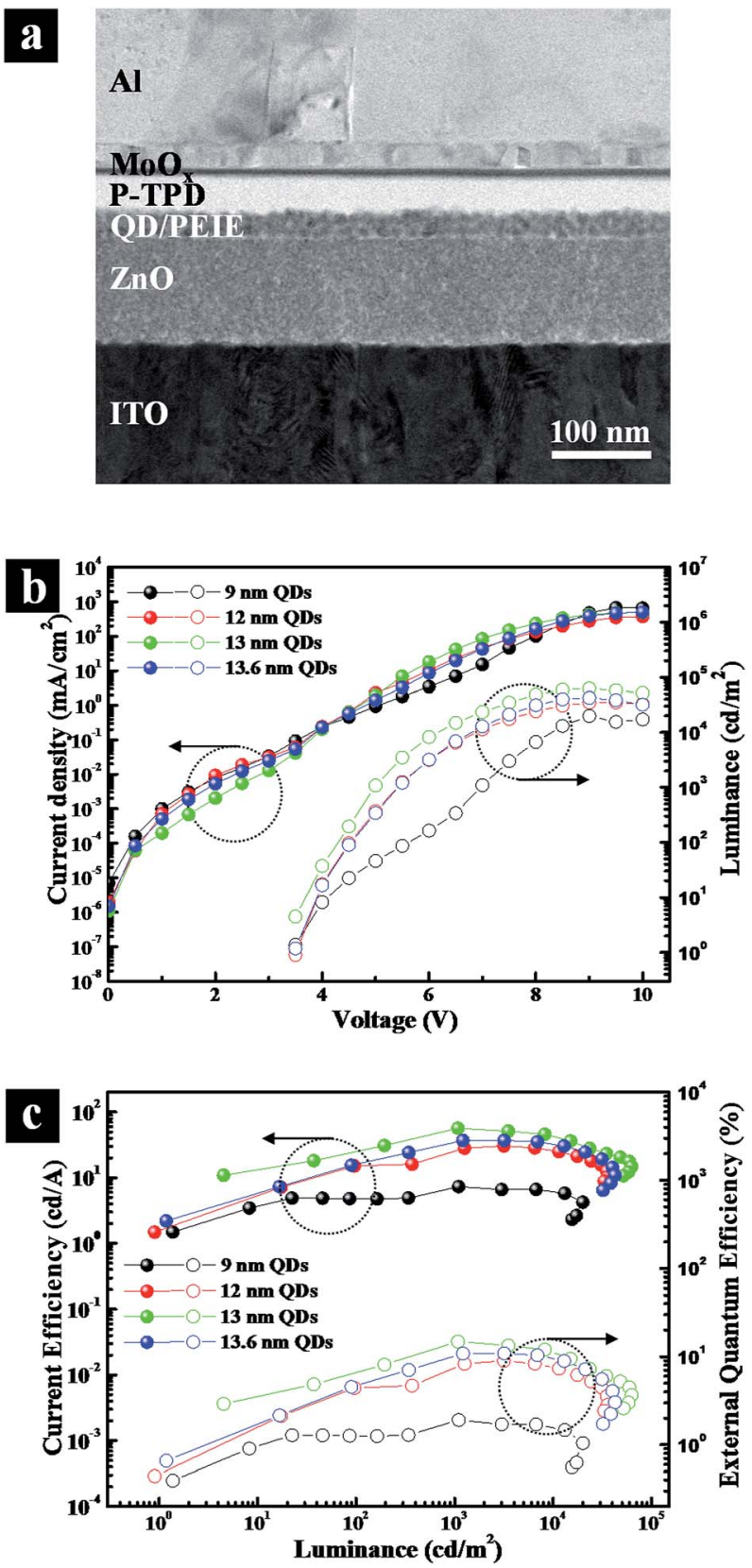

Fig. 5 Device performance was affected by QD size with QD EML. (a) Cross-sectional transmission electron microscopy image of QLED; (b) current density-voltage-luminance $(J-V-L)$ characteristics; (c) current efficiency and external quantum efficiency characteristics.

UV/ozone irradiation $\left(\mathrm{O}_{2} \rightarrow 2 \mathrm{O}^{-} ; \mathrm{O}^{-}+\mathrm{O}_{2} \rightarrow \mathrm{O}_{3}\right)$ in intervals of 30 seconds. The figure shows that the PL intensity decreases to $61 \%$ of the initial PL intensity after 30 seconds of exposure in the absence of the outer shell (A-QDs). After $240 \mathrm{~s}$ exposure, the intensity is only $30 \%$ of the initial phase of the irradiation. In comparison, the intensity also decreases $12 \%$ in the initial phase of the irradiation even for the QDs with the outer shell (AS-QDs) and is more stable against oxidation. At the end of test, AS-QDs still maintains $65 \%$ of its initial PL intensity, which is 1.5 times higher than the initial PL intensity of A-QDs and
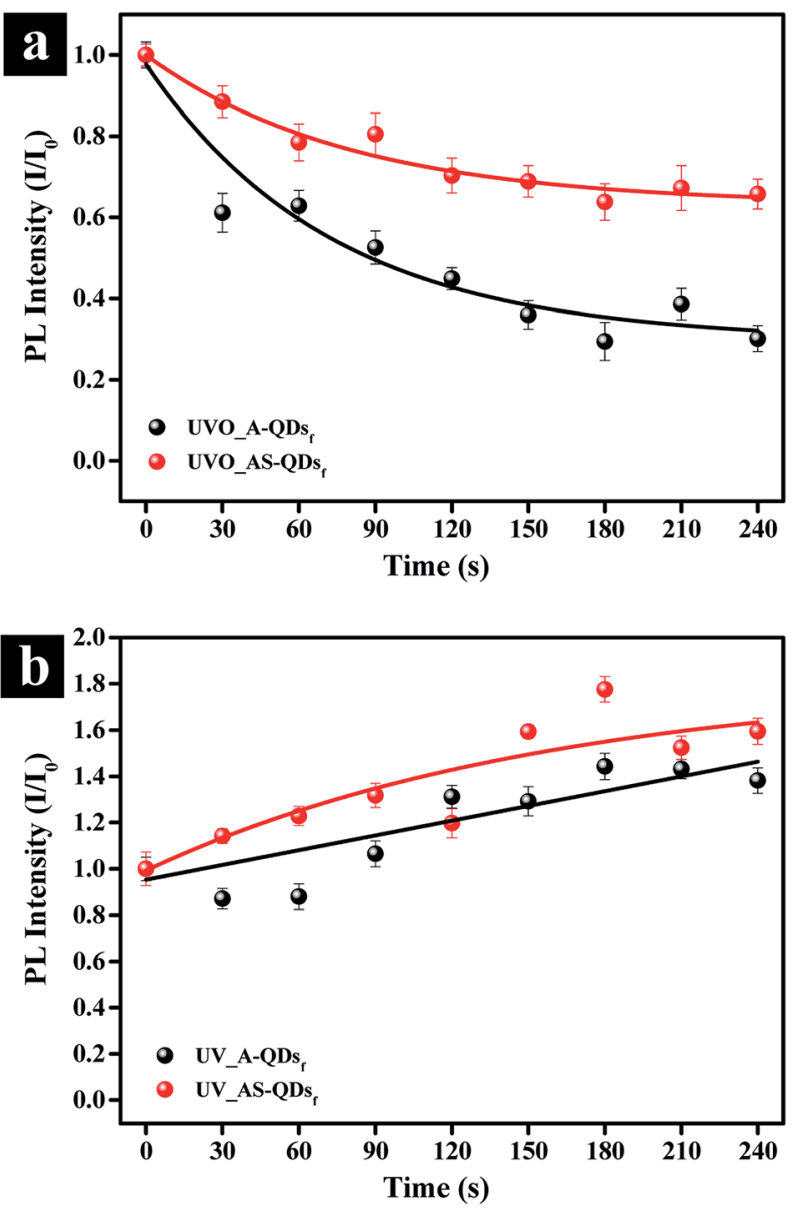

Fig. 6 Evolution of the photo-stimulated degradation stability of A- and AS-QDs. (a) UV irradiation exposure $(185 \mathrm{~nm}, 254 \mathrm{~nm}$, $180 \mathrm{~mW} \mathrm{~cm}^{-2}$ ) in $\mathrm{O}_{2}$ environment. (b) UV irradiation exposure (185 nm, $254 \mathrm{~nm}, 180 \mathrm{~mW} \mathrm{~cm}^{-2}$ ) in air environment.

5 times higher than the final PL intensity of A-QDs (Fig. 6a and $\mathrm{S} 2 \mathrm{a} \dagger)$. This result indicates that the outer shell as a protective barrier efficiently decreases the probability of surface oxidation and resists the quenching.

The effect of UV irradiation alone in the absence of oxygen or ozone $\left(\mathrm{O}_{2}\right.$ or $\left.\mathrm{O}_{3}\right)$ was investigated. UV exposure in the air (Fig. 6b) was achieved by sealing samples to isolate them from external oxygen or ozone (Fig. 6a). The results in Fig. 6b and $\mathrm{S} 2 \mathrm{~b} \uparrow$ show an increase in the PL of both two kinds of QDs. The enhancement in the PL of QDs can be attributed to the photoactivation of QDs with UV illumination and the assistance of a little oxygen in the air that passivates the surface defects. ${ }^{27}$ In conclusion, UV irradiation in air (little $\mathrm{O}_{2}$ ) can enhance the PL of QDs by photoactivation phenomenon; but the UVO reduces the PL of QDs. A relatively thick inorganic shell can effectively eliminate the probability of photo-oxidation and improve the photostability. There was no blue-shift in the emission spectrum of QDs (Fig. S2 $\dagger$ ), which indicates that the size of QDs is unchanged.

Long-term thermal stability is also examined by subjecting the QD films to high temperature and high humidity. When the 
QD films are heated at $120{ }^{\circ} \mathrm{C}$ in $\mathrm{N}_{2}$ glove box (Fig. 7a), the PL intensity of A-QD practically disappears after $360 \mathrm{~min}$.

However, the intensity of AS-QD reaches a plateau of $60 \%$ after $250 \mathrm{~min}$ and do not decrease much thereafter. The two types of QDs are also subjected to $85{ }^{\circ} \mathrm{C}, 85 \%$ relative humidity $(\mathrm{RH})$ conditions in air. Fig. 7b shows that with time increasing, the AS-QD film has a little fluctuation in relative PL intensity but
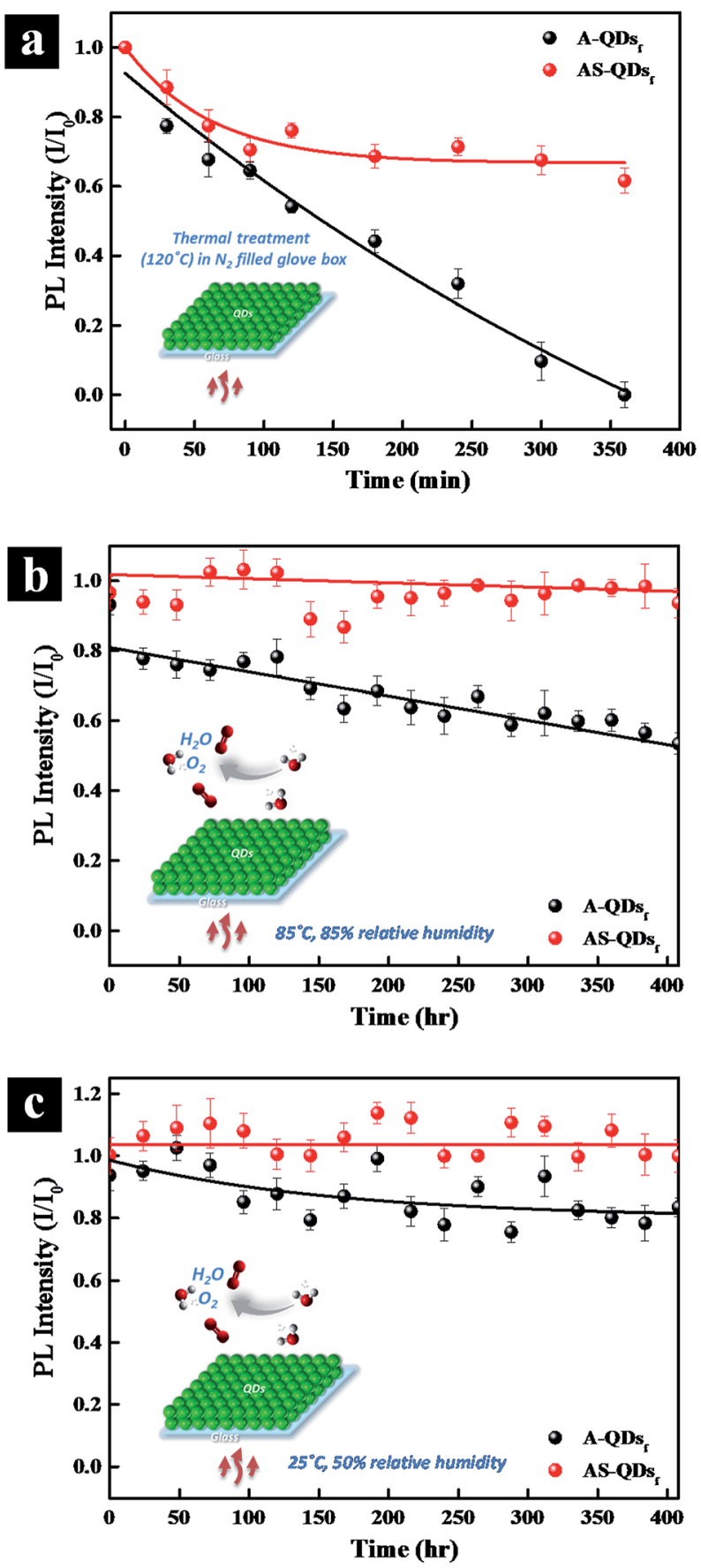

Fig. 7 Evolution of the long-term ( $400 \mathrm{~h})$ thermal stability of A- and AS-QDs. (a) Thermal treatment $\left(120^{\circ} \mathrm{C}\right)$ in $\mathrm{N}_{2}$ filled glove box; (b) thermal treatment under $85^{\circ} \mathrm{C}, 85 \%$ relative humidity conditions in air. (c) Thermal treatment under $25^{\circ} \mathrm{C}, 50 \%$ relative humidity conditions in air. do not show any apparent adverse effects over the period of $400 \mathrm{~h}$. On the other hand, the relative PL intensity of A-QD film decreases steadily over the period of time. It is notable that only a 7\% loss in PL occurred even after $400 \mathrm{~h}$ of exposure of AS-QDs to harsh thermal conditions. Fig. 7c examines the stability of the two different QD films under the conditions of $25{ }^{\circ} \mathrm{C}$ and $50 \% \mathrm{RH}$ over a period of $400 \mathrm{~h}$. Compared to the harsher conditions of $85{ }^{\circ} \mathrm{C}$ and $85 \% \mathrm{RH}$, the PL intensity of the AS-QD film is much more stable, the value being almost invariant with time. These results clearly indicate that the film of AS-QDs is more stable against moisture and oxygen compared to that of A-QDs. The outer shell provides an excellent physical barrier and protects the core of QDs against high temperature, oxygen, and moisture, leading to the long term thermal stability of the PL of the QDs.

\section{Conclusion}

In conclusion, we have found that the absolute PL QY of QDS can be increased to $88 \%$ by properly selecting the thickness of the outer shell that coats the alloyed, graduated core/shell QDs. When these QDs with the optimal thickness were used in a QLED, more than a 7 fold increase in the external quantum efficiency resulted compared to the QDs without the outer shell, reflecting the substantial improvement in PL QY due to the outer shell. The AS-QDs with the outer shell have been found to resist degradation when exposed to UV in oxygen or ozone environment. They also exhibit long-term stability even after $400 \mathrm{~h}$ of exposure to high temperature and high humidity. Removal of non-radiative recombination sources and presence of a physical barrier, made possible by the alloyed, graded core/ shell with outer shell of optimal thickness, is believed to be responsible for the excellent performance.

\section{Conflicts of interest}

There are no conflicts to declare.

\section{Acknowledgements}

This work was financially supported by the Basic Science Research Program through the National Research Foundation of Korea (NRF) funded by the Ministry of Education, Science and Technology (NRF-2015R1A2A2A01003520). This research was also supported by the Ministry of Science, ICT and Future Planning (MSIP), Korea, under the Information Technology Research Center (ITRC) support program (IITP-2016-H8501-161009) supervised by the Institute for Information \& Communications Technology Promotion (IITP).

\section{Notes and references}

1 W. K. Bae, S. Brovelli and V. I. Klimov, MRS Bull., 2013, 38, 721.

2 B. Dabbousi, M. Bawendi, O. Onitsuka and M. Rubner, Appl. Phys. Lett., 1995, 66, 1316. 
3 S. Coe, W.-K. Woo, M. Bawendi and V. Bulović, Nature, 2002, 420, 800.

4 D. Kim, Y. Fu, S. Kim, W. Lee, K.-H. Lee, H. K. Chung, H.-J. Lee, H. Yang and H. Chae, ACS Nano, 2017, 11, 1982.

5 O. E. Semonin, J. M. Luther, S. Choi, H.-Y. Chen, J. Gao, A. J. Nozik and M. C. Beard, Science, 2011, 334, 1530.

6 X. Wang, G. I. Koleilat, J. Tang, H. Liu, I. J. Kramer, R. Debnath, L. Brzozowski, D. A. R. Barkhouse, L. Levina and S. Hoogland, Nat. Photonics, 2011, 5, 480.

7 M. Bruchez, M. Moronne, P. Gin, S. Weiss and A. P. Alivisatos, Science, 1998, 281, 2013.

8 W. C. Chan and S. Nie, Science, 1998, 281, 2016.

9 X. Gao, Y. Cui, R. M. Levenson, L. W. Chung and S. Nie, Nat. Biotechnol., 2004, 22, 969.

10 H. Y. Yang, Y. Fu, M.-S. Jang, Y. Li, J. H. Lee, H. Chae and D. S. Lee, ACS Appl. Mater. Interfaces, 2016, 8, 35021.

11 L. H. Qu and X. G. Peng, J. Am. Chem. Soc., 2002, 124, 2049. 12 R. Xie, U. Kolb, J. Li, T. Basché and A. Mews, J. Am. Chem. Soc., 2005, 127, 7480.

13 W. K. Bae, J. Kwak, J. W. Park, K. Char, C. Lee and S. Lee, Adv. Mater., 2009, 21, 1690.

14 S. K. Panda, S. G. Hickey, C. Waurisch and A. Eychmüller, J. Mater. Chem., 2011, 21, 11550.

15 H. Shen, S. Wang, H. Wang, J. Niu, L. Qian, Y. Yang, A. Titov, J. Hyvonen, Y. Zheng and L. S. Li, ACS Appl. Mater. Interfaces, 2013, 5, 4260.

16 A. Wang, H. Shen, S. Zang, Q. Lin, H. Wang, L. Qian, J. Niu and L. Song Li, Nanoscale, 2015, 7, 2951.

17 D. Kim, Y. Fu, J. Kim, K. H. Lee, H. Kim, H. Yang and H. Chae, Nanotechnology, 2016, 27, 245203.

18 Y. Fu, D. Kim, H. Moon, H. Yang and H. Chae, J. Mater. Chem. C, 2017, 5, 522.

19 X. Zhong, M. Han, Z. Dong, T. J. White and W. Knoll, J. Am. Chem. Soc., 2003, 125, 8589.
20 B. C. Fitzmorris, Y. C. Pu, J. K. Cooper, Y. F. Lin, Y. J. Hsu, Y. Li and J. Z. Zhang, ACS Appl. Mater. Interfaces, 2013, 5, 2893.

21 A. R. Clapp, I. L. Medintz and H. Mattoussi, ChemPhysChem, 2006, 7, 47.

22 K.-H. Lee, J.-H. Lee, H.-D. Kang, B. Park, Y. Kwon, H. Ko, C. Lee, J. Lee and H. Yang, ACS Nano, 2014, 8, 4893.

23 B. N. Pal, Y. Ghosh, S. Brovelli, R. Laocharoensuk, V. I. Klimov, J. A. Hollingsworth and H. Htoon, Nano Lett., 2012, 12, 331.

24 S. Xu, H. Shen, C. Zhou, H. Yuan, C. Liu, H. Wang, L. Ma and L. S. Li, J. Phys. Chem. C, 2011, 115, 20876.

25 S. Jun and E. Jang, Angew. Chem., 2013, 52, 679.

26 Y. Yang, Y. Zheng, W. Cao, A. Titov, J. Hyvonen, J. R. Manders, J. Xue, P. H. Holloway and L. Qian, Nat. Photonics, 2015, 9, 259.

27 C. Carrillo-Carrion, S. Cardenas, B. M. Simonet and M. Valcarcel, Chem. Commun., 2009, 5214.

28 W. K. Bae, J. Kwak, J. W. Park, K. Char, C. Lee and S. Lee, Adv. Mater., 2009, 21, 1690.

29 M. D. Ho, D. Kim, N. Kim, S. M. Cho and H. Chae, ACS Appl. Mater. Interfaces, 2013, 5, 12369.

30 H. Kim, J. Y. Han, D. S. Kang, S. W. Kim, D. S. Jang, M. Suh, A. Kirakosyan and D. Y. Jeon, J. Cryst. Growth, 2011, 326, 90.

31 J. Kwak, J. Lim, M. Park, S. Lee, K. Char and C. Lee, Nano Lett., 2015, 15, 3793.

32 J. Lim, B. G. Jeong, M. Park, J. K. Kim, J. M. Pietryga, Y. S. Park, V. I. Klimov, C. Lee, D. C. Lee and W. K. Bae, Adv. Mater., 2014, 26, 8034.

33 W. K. Bae, Y. S. Park, J. Lim, D. Lee, L. A. Padilha, H. McDaniel, I. Robel, C. Lee, J. M. Pietryga and V. I. Klimov, Nat. Commun., 2013, 4, 2661.

34 V. I. Klimov, Annu. Rev. Condens. Matter Phys., 2014, 5, 285. 35 B. N. Pal, Y. Ghosh, S. Brovelli, R. Laocharoensuk, V. I. Klimov, J. A. Hollingsworth and H. Htoon, Nano Lett., 2011, 12, 331. 